\title{
Antitumor Properties of Irinotecan-Containing Nanoparticles Prepared Using Poly(DL-lactic acid) and Poly(ethylene glycol)-block-poly(propylene glycol)-block-poly(ethylene glycol)
}

\author{
Hiraku ONISHI, ${ }^{*, a}$ Yoshiaki MACHIDA, ${ }^{b}$ and Yoshiharu MACHIDA ${ }^{a}$ \\ ${ }^{a}$ Department of Drug Delivery Research, Hoshi University; 2-4-41 Ebara, Shinagawa-ku, Tokyo 142-8501, Japan: and \\ ${ }^{b}$ Department of Pharmacy, Hachioji Digestive Organs Hospital; 177 Yorozu-cho, Hachioji, Tokyo 192-0903, Japan. \\ Received May 24, 2002; accepted September 17, 2002
}

\begin{abstract}
Irinotecan-containing nanoparticles (NP) were prepared by coprecipitation with addition of water to acetone solution of poly(DL-lactic acid), poly(ethylene glycol)-block-poly(propylene glycol)-block-poly(ethylene glycol) and irinotecan, and subsequent evaporation of organic solvent. NP were purified by gel filtration and used for experiments after condensation by evaporation. The obtained NP showed the drug content of $4.5 \%(w / w)$ and the mean particle diameter of $118 \mathrm{~nm}$ with the particle diameter distribution between $80-210 \mathrm{~nm}$. When the antitumor effect was examined at a repeated dose of $20 \mathrm{mg}$ irinotecan eq/ $\mathrm{kg}$ for $3 \mathrm{~d}(3 \times 20 \mathrm{mg} / \mathrm{kg})$ using mice bearing Sarcoma 180 subcutaneously, only NP suppressed tumor growth significantly. After i.v. injection in rats, NP maintained irinotecan plasma concentration longer than CPT-11 aqueous solution. The present nanoparticle formation is suggested as a possibly useful dosage form of irinotecan against solid tumor.
\end{abstract}

Key words nanoparticle; poly(DL-lactic acid); irinotecan; poly(ethylene glycol)-block-poly(propylene glycol)-block-poly(ethylene glycol); antitumor effect; plasma concentration

Camptothecin, an alkaloid isolated from the Chinese tree Camptotheca acuminata, was found as a new type of antitumor agent. It inhibited the activity of topoisomerase I, and showed S-phase specific cytotoxicity. ${ }^{1-4)}$ A semi-synthetic derivative of camptothecin, 7-ethyl-10-hydroxycamptothecin (SN-38), is highly potent in vitro. ${ }^{2,5)}$ However, SN-38 does not always exhibit good efficacy in vivo due to its pharmacokinetic characteristics. ${ }^{5,6)}$ Irinotecan, which is a prodrug of SN-38, shows good efficacy against various tumors in vivo, ${ }^{7-9)}$ and exhibits a higher efficacy in vivo than SN-38. ${ }^{6}$ ) This high potency of irinotecan is considered due to its good retention in the body and to appropriate regeneration of SN38 there. ${ }^{5)}$ However, irinotecan severely suppresses bone marrow cells and causes severe diarrhea. ${ }^{10-12)}$ Therefore, dosage forms have been investigated in an attempt to improve its antitumor characteristics. Since the antitumor effect of camptothecin is generally time-dependent, $, 2,4)$ dosage forms which increase retention of the drug in the body may improve the efficacy of irinotecan. Microspheres, showing prolonged release of irinotecan, were developed using poly(DL-lactic acid) (PLA) or poly(DL-lactic acid-co-glycolic acid) (PLGA). ${ }^{13,14)}$ These microspheres, administered intraperitoneally, exhibited an equivalent or higher effect against P388 leukemia inoculated into the peritoneal cavity, but were less effective against Sarcoma 180 solid tumor inoculated subcutaneously. ${ }^{13)}$ These findings suggested that the simple prolonged release system of irinotecan is not necessarily satisfactory for treatment of a tumor distant from the administration site, probably because of insufficient supply of the active form, SN-38. ${ }^{14)}$ Therefore, development of dosage forms which give a high drug concentration around tumor cells or possess an ability to home to the tumor site is considered necessary. Nanoparticles with a diameter of less than $200 \mathrm{~nm}$ can accumulate in diseased parts with highly permeable blood vessels, such as solid tumor, ${ }^{15)}$ so preparation of such nanoparticles was attempted in this study. Further, poly(ethylene glycol)-block-poly(propylene glycol)-block- poly(ethylene glycol) has recently been reported to be a very available stabilizing agent for nanoparticle suspension, even under in vivo conditions. ${ }^{16)}$ Thus, in this study, preparation of PLA nanoparticles containing irinotecan with poly(ethylene glycol)-block-poly(propylene glycol)-block-poly(ethylene glycol) as a stabilizing agent was attempted, and the obtained nanoparticles were examined for particle characteristics, antitumor effect against Sarcoma 180 solid tumor and pharmacokinetics.

\section{MATERIALS AND METHODS}

Materials Poly(DL-lactic acid) (PLA; MW 10000) was purchased from Wako Pure Chemical Industries, Ltd. (Osaka, Japan). Poly(ethylene glycol)-block-poly(propylene glycol)-block-poly(ethylene glycol) (PEG-PPG-PEG; MW $8400,80 \%(\mathrm{w} / \mathrm{w})$ ethylene glycol) was purchased from Aldrich Chemical Company, Inc. (Milwaukee, U.S.A.). Irinotecan hydrochloride (CPT-11) and 7-ethyl-10-hyroxycamptothecin (SN-38) were generously supplied by Yakult Honsha, Co., Ltd. (Tokyo, Japan). All other chemicals were of reagent grade.

Animals Male Wistar rats weighing 190-210g (6 weeks old) and male ddY mice weighing 24-28 g (6 weeks old) were purchased from Tokyo Laboratory Animals Science Co., Ltd. (Tokyo, Japan). Soon after purchase, they were used for the animal experiments. All animal experiments conformed to the Guidelines for Animal Experimentation of Hoshi University.

Preparation of Nanoparticles CPT-11 was dissolved in water, the solution $\mathrm{pH}$ was adjusted to 8.5 by sodium bicarbonate, and the suspension obtained was extracted with chloroform. The chloroform layer was collected and dried over sodium sulfate. After removal of sodium sulfate by filtration, the solvent was evaporated. The pale yellow powder remaining was used as a very chloroform-soluble form in the preparation of nanoparticles (NP). This drug powder (irinotecan) 
$(20 \mathrm{mg})$, PLA (50 mg) and PEG-PPG-PEG (30 mg) were dissolved in $3 \mathrm{ml}$ of acetone. Water $(3 \mathrm{ml})$ was added to the solution, and acetone was evaporated at $35^{\circ} \mathrm{C}$ using a rotary evaporator for $1 \mathrm{~h}$ to produce NP. NP were then separated from the free drug with a Sephadex G-50 column $(3 \mathrm{~cm}$ (inner diameter) $\times 14 \mathrm{~cm}$ (length)) using water as an elution solvent. The obtained NP aqueous suspension was condensed into approximately $2 \mathrm{ml}$, and used in the following experiments.

Particle Characteristics The particle size distribution of NP was measured by dynamic light scattering using an ELS-800 (Otsuka Electronics Co., Ltd.) after appropriate dilution of NP aqueous suspension with water. The yield of NP was calculated as the ratio of the amount of recovered NP to the total amount of (added PLA+added PEG-PPG-PEG+ added irinotecan) or (added PLA+added irinotecan). After a certain volume of NP aqueous suspension was dried under $\mathrm{N}_{2}$ gas at $40^{\circ} \mathrm{C}$, the residue was dissolved in chloroform, and measured spectrophotometrically at $365 \mathrm{~nm}$ to determine the amount of irinotecan contained in NP. The drug recovery was calculated as the ratio of the amount of recovered irinotecan to the amount of added irinotecan.

In Vivo Antitumor Effect against Sarcoma 180 Solid Tumor Sarcoma 180 cells were maintained by weekly transplantation of $1 \times 10^{7}$ cells suspended in Hanks' balanced solution $(0.1 \mathrm{ml})$ into the peritoneal cavity of a ddY mouse. In the antitumor test, $1 \times 10^{7}$ of Sarcoma 180 cells suspended in Hanks' balanced solution $(0.1 \mathrm{ml})$, obtained in the above transplantation, were inoculated subcutaneously at the axillary region of each new ddY mouse. NP aqueous suspension or CPT-11 aqueous solution $(0.2-0.3 \mathrm{ml})$ was injected intravenously into the tail vein of each mouse repeatedly at the dose of $20 \mathrm{mg}$ irinotecan eq/ $\mathrm{kg}$ at 7,8 and $9 \mathrm{~d}$ after inoculation, i.e. $20 \times 3 \mathrm{mg}$ irinotecan eq $/ \mathrm{kg}(n=5)$. As a control, saline alone of similar volume was injected on the same schedule. Tumor volume $\left(V, \mathrm{~cm}^{3}\right)$ was calculated from the length of the longest axis $(L, \mathrm{~cm})$ and the length of the vertical axis to the longest one, width, $(W, \mathrm{~cm})$ by the following equation ${ }^{17)}$ :

$$
V=L \times W^{2} / 2
$$

The tumor volume observed immediately prior to the first administration was defined as the initial tumor volume $\left(V_{0}\right)$; tumor growth was calculated as the ratio of $V$ to $V_{0}$.

At the same time, change in body weight of each mouse was measured as an index of toxic side effect.

Plasma Concentration after I.v. Administration of Nanoparticles Male Wistar rats were anesthetized by i.p. injection of urethane solution in saline at $0.9 \mathrm{~g} / 4 \mathrm{ml} / \mathrm{kg}$, and fixed on the back. NP aqueous suspension or CPT-11 aqueous solution $(0.2-0.3 \mathrm{ml})$ was intravenously administered to each rat at $3 \mathrm{mg}$ irinotecan eq $/ \mathrm{kg}$ from the jugular vein. At appropriate times, blood $(0.5 \mathrm{ml})$ was withdrawn using a heparinized syringe and centrifuged at $15000 \mathrm{rpm}$ for $30 \mathrm{~s}$ to obtain the plasma. Immediately after that, $0.15 \mathrm{M}$ aqueous phosphoric acid $(0.2 \mathrm{ml})$ was added to the plasma $(0.1 \mathrm{ml})$ and mixed vigorously. Irinotecan was extracted with chloroform $(0.3 \mathrm{ml})$. After centrifugation of the mixture at 3000 $\mathrm{rpm}$ for $10 \mathrm{~min}, 20 \mu \mathrm{l}$ of the chloroform layer was directly injected on high performance liquid chromatography (HPLC) to determine the concentration of irinotecan. This operation gave the total concentration of free and incorporated irinotecan for NP, and the concentration of free irinotecan for CPT11 aqueous solution.

The HPLC analysis was performed at $25^{\circ} \mathrm{C}$. A Shimadzu LC-6AD apparatus equipped with a fluorescence detector Shimadzu RF-10AXL in which excitation and emission wavelengths were set at 355 and $515 \mathrm{~nm}$, respectively, was used. A Nova-Pack $5 \mathrm{C}_{18}(4.6 \mathrm{~mm}$ in inner diameter $\times 150 \mathrm{~mm}$ in length) was used as an analytical column. The mixture of $0.075 \mathrm{M}$ ammonium acetate, $\mathrm{pH} 6.4$, and acetonitrile $(78: 22$, $\mathrm{v} / \mathrm{v}$ ) containing $5 \mathrm{~mm}$ tetrabutylammonium dihydrogen phosphate was used as a mobile phase, and the flow rate was set at $1.5 \mathrm{ml} / \mathrm{min}$.

The pharmacokinetic parameters were calculated by the following equations:

$$
\begin{aligned}
A U C & =\int_{0}^{\infty} C \mathrm{p} \mathrm{d} t / A U C \\
M R T & =\int_{0}^{\infty}(C \mathrm{p} \times t) \mathrm{d} t / A U C \\
V R T & =\int_{0}^{\infty}\left\{\left(C \mathrm{p} \times(t-M R T)^{2}\right\} \mathrm{d} t / A U C\right.
\end{aligned}
$$

where $C \mathrm{p}$ meant the plasma concentration at time $t$. The $C \mathrm{p}$ at $t=0$ was calculated by extrapolation using three points in initial periods, and extrapolation of the $C \mathrm{p}$ curve to $t=\infty$ was performed by assuming that the plasma concentrations in the latter phase followed the mono-exponential decline. The trapezoidal method was used for calculation of each parameter.

Statistical Analysis Statistical analysis was performed using the unpaired $t$-test. Significant difference was set as $p<0.05$.

\section{RESULTS}

Particle Characteristics Obtained NP had a mean particle diameter of $118 \mathrm{~nm}$ with particle diameter distribution between $80-210 \mathrm{~nm}$. This size is adequate for avoidance of entrapment by the reticuloendothelium, leading to passive targeting to diseased parts with highly permeable blood vessels. ${ }^{15)}$ The yields calculated by (amount of recovered NP)/ (total added amount of PLA, PEG-PPG-PEG and irinotecan: $100 \mathrm{mg}$ ) and (amount of recovered NP)/(total added amount of PLA and irinotecan: $70 \mathrm{mg}$ ) were 50 and $72 \%(\mathrm{w} / \mathrm{w})$, respectively. Further, the drug content and drug recovery were calculated to be 4.5 and $11 \%(\mathrm{w} / \mathrm{w})$, respectively.

\section{In Vivo Antitumor Effect against Sarcoma 180 Solid} Tumor The growth rates of Sarcoma 180 solid tumor inoculated subcutaneously were compared among NP aqueous suspension, CPT-11 aqueous solution and control (saline) (Fig. 1). Significant suppression of tumor growth was observed in NP aqueous suspension, while CPT-11 aqueous solution showed no marked difference from control. The change in mean body weight was used as an index of toxic side effects (Fig. 2). Body weight was not reduced from the initial body weight, and the mean body weight was larger in the order of control $>$ CPT-11 aqueous solution $>\mathrm{NP}$ aqueous suspension during the observation period. Diarrhea, known to be one of the toxic side effects, was not observed in either NP aqueous suspension or CPT-11 aqueous solution. 


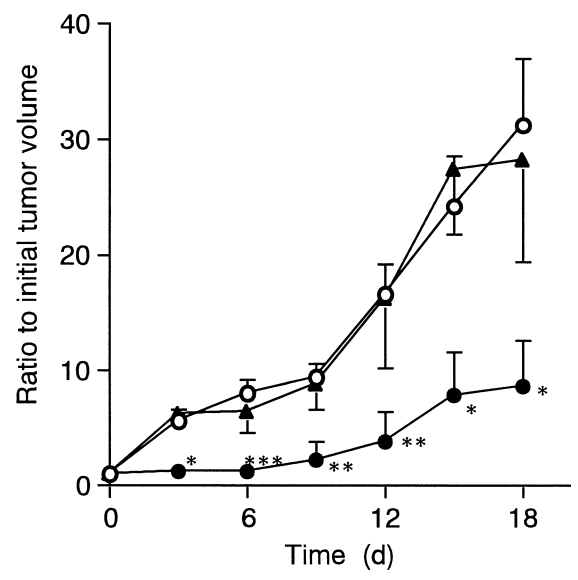

Fig. 1. Growth-Inhibitory Effect of NP Aqueous Suspension (-) and CPT-11 Aqueous Solution ( $\mathbf{\Lambda})$ against Sarcoma 180 Solid Tumor after I.v. Administration

Control is shown by open circles $(\bigcirc)$. Each daily intravenous injection was performed at the dose of $20 \mathrm{mg}$ irinotecan eq $/ \mathrm{kg}$ at $7-9 \mathrm{~d}$ after s.c. inoculation, i.e. $20 \times 3$ irinotecan eq $/ \mathrm{kg}$. Each point represents the mean \pm S.E. $(n=5) . * p<0.05, * * p<0.01$, $* * * p<0.001$ vs. control.

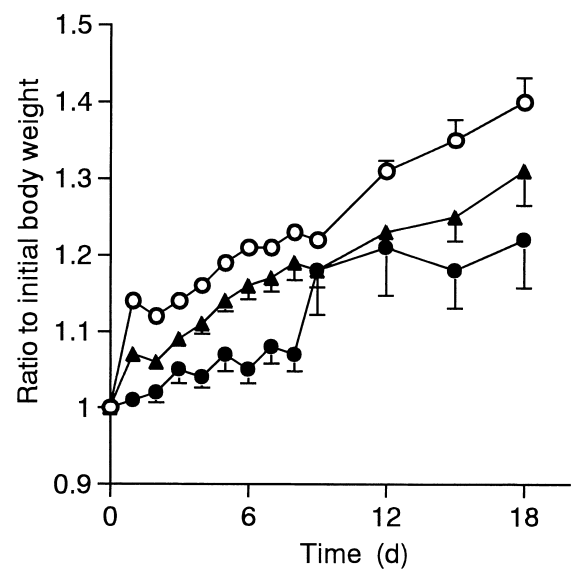

Fig. 2. Change in Body Weight of Sarcoma 180-Bearing Mice after I.v. Administration of NP Aqueous Suspension (-) and CPT-11 Aqueous Solution $(\boldsymbol{\Lambda})$

The data were obtained in mice described in Fig. 1. Control is shown by open circles (O). Each daily intravenous injection was performed at the dose of $20 \mathrm{mg}$ irinotecan $\mathrm{eq} / \mathrm{kg}$ at $7-9 \mathrm{~d}$ after s.c. inoculation, i.e. $20 \times 3$ irinotecan $\mathrm{eq} / \mathrm{kg}$. Each point represents the mean \pm S.E. $(n=5)$.

Plasma Concentration-Time Profiles after I.v. Administration Plasma concentration-time profiles were examined after i.v. administration of NP aqueous suspension and CPT-11 aqueous solution. For NP aqueous suspension, the result showed the total (free and NP-incorporated) concentration of irinitecan. With i.v. administration of CPT-11 aqueous solution ( $3 \mathrm{mg}$ irinotecan eq $/ \mathrm{kg}$ ), the plasma concentration was $0.70 \mu \mathrm{g} / \mathrm{ml}$ at $1 \mathrm{~h}$ post-injection, and the plasma concentration decreased mono-exponentially to $0.02 \mu \mathrm{g} / \mathrm{ml}$ at $8 \mathrm{~h}$ post-injection. When NP aqueous suspension was administered intravenously, the plasma concentrations were 0.46 and $0.18 \mu \mathrm{g} / \mathrm{ml}$ at 1 and $2 \mathrm{~h}$ post-injections, respectively, which were lower than those in CPT-11 aqueous solution. However, the plasma concentration tended to be higher in NP aqueous suspension than in CPT-11 aqueous solution after $4 \mathrm{~h}$ postadministration (Fig. 3). The pharmacokinetic parameters are described in Table 1. Each parameter was larger in NP aque-

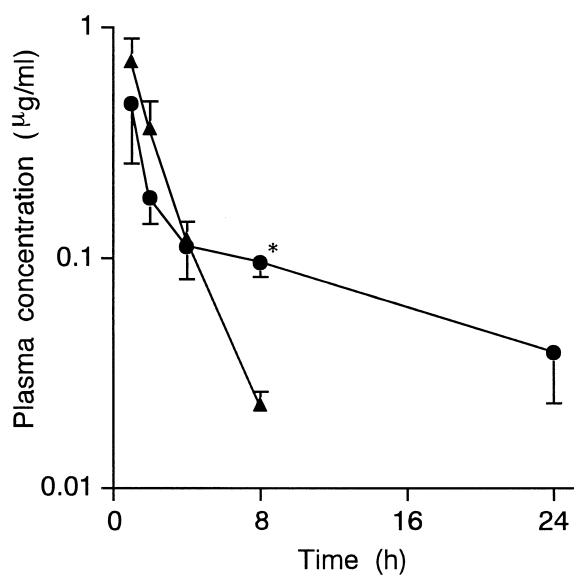

Fig. 3. Plasma Concentration-Time Profiles of Irinotecan after I.v. Administration of NP Aqueous Suspension (O) and CPT-11 Aqueous Solution (A) to Rats

Each preparation was intravenously injected at the dose of $3 \mathrm{mg}$ irinotecan eq $/ \mathrm{kg}$ to rats. Each point represents the mean \pm S.E. $(n=4-5)$. $* p<0.05$ vs. CPT- 11 aqueous solution.

Table 1. Pharmacokinetic Parameters in I.v. Administration of CPT-11 Aqueous Solution and NP Aqueous Suspension

\begin{tabular}{lccc}
\hline \hline \multicolumn{1}{c}{ Dosage form } & $\begin{array}{c}A U C \\
(\mu \mathrm{g} \cdot \mathrm{h} / \mathrm{ml})\end{array}$ & $\begin{array}{c}M R T \\
(\mathrm{~h})\end{array}$ & $\begin{array}{c}V R T \\
\left(\mathrm{~h}^{2}\right)\end{array}$ \\
\hline CPT-11 aqueous solution & $2.12 \pm 0.35$ & $2.03 \pm 0.34$ & $5.29 \pm 1.37$ \\
NP aqueous suspension & $3.16 \pm 0.51$ & $11.92 \pm 1.81 *$ & $228.62 \pm 69.41^{*}$
\end{tabular}

The results are expressed as the mean \pm S.E. ( $n=5$ for CPT- 11 aqueous solution; $n=4$ for NP aqueous suspension). $*: p<0.05 v s$. CPT-11 aqueous solution.

ous suspension, with $M R T$ and $V R T$ being significantly greater. The plasma concentration of $\mathrm{SN}-38$ was observed to be more than $7 \mathrm{ng} / \mathrm{ml}$ at $8 \mathrm{~h}$ post-administration in NP aqueous suspension by a similar HPLC method.

\section{DISCUSSION}

Nanoparticles with a particle diameter of less than $200 \mathrm{~nm}$ and a PEG-coated surface have been found to well avoid the entrapment by the reticuloendothelium and to well leak in diseased parts with highly permeable blood vessels, resulting in passive targeting to the diseased parts ${ }^{15)}$; this is known as an enhanced permeability and retention (EPR) effect. ${ }^{18)}$ The present NP were prepared using PEG-PPG-PEG as a stabilizing agent of suspension, and the particle size was found to be adequate for such effect. Further, the yield and drug content of NP were fairly good. The particle characteristics in other formulations will be a future subject.

The antitumor effects against Sarcoma 180 solid tumor and plasma concentration-time profiles were compared between CPT-11 aqueous solution and NP aqueous suspension. CPT-11 aqueous solution was not effective, but NP aqueous suspension significantly suppressed tumor growth (Fig. 1). On the contrary, simple prolonged release microspheres reported previously were less effective against solid tumor than CPT-11 aqueous solution. ${ }^{13,14)}$ Therefore, NP were considered to possess functions different from the previous microspheres. Body weight decrease, a toxic side effect, was not observed in the present examination (Fig. 2). Diarrhea, a typical toxic side effect, was not observed in any group. Thus, the 
present NP were suggested to be possibly useful against solid tumor distant from the administration site. The plasma concentration (free plus NP-loaded irinotecan)-time profiles tended to maintain the plasma levels of irinotecan longer in NP aqueous suspension than in CPT-11 aqueous solution (Fig. 3), indicating that NP could cause better retention of the drug in the plasma. The $A U C, M R T$ and $V R T$ values were larger in NP aqueous suspension than in CPT-11 aqueous solution, suggesting that NP themselves would circulate better and longer in the blood stream (Table 1). The prolonged retention of NP in the plasma was considered to be due to avoidance of the entrapment by the reticuloendothelium, and NP might be accumulated in the tumor tissue based on the EPR effect. ${ }^{18)}$ Although the plasma concentration of SN-38 was not checked in detail, SN-38 was observed at the concentration of more than $7 \mathrm{ng} / \mathrm{ml}$ at $8 \mathrm{~h}$ after i.v. administration of NP aqueous suspension (data not shown), indicating a fairly long retention of $\mathrm{SN}-38$ in this formation. These plasma concentration data appear to support better therapeutic potency of the NP aqueous suspension. In conclusion, the present NP system prepared with PLA, PEG-PPG-PEG and irinotecan is suggested to be possibly useful against solid tumor when it is administered intravenously in an aqueous suspension form. However, examination on tumor accumulation of the drug is needed to elucidate the mechanism of NP efficacy.

\section{REFERENCES}

1) Nagata H., J. Aichi Med. Univ. Assoc., 15, 683-699 (1987).
2) Kawato Y., Aonuma M., Hirota Y., Kuga H., Sato K., Cancer Res., 51, 4187-4191 (1991).

3) Okada K., Ando T., Antibiot. Chemother., 9, 221-228 (1993).

4) Inaba M., Abstract of Research Works for the General Research A of Subject Number 05302061 with a Grant-in-Aid for Scientific Research from the Ministry of Education, Science, Sports and Culture of Japan, 1994, pp. 57-61.

5) Kaneda N., Nagata H., Furuta T., Yokokura T., Cancer Res., 50, 1715-1720 (1990).

6) Tsuruo T., Matsuzaki T., Matsushita M., Saito H., Yokokura T., Cancer Chemother. Pharmacol., 21, 71-74 (1988).

7) Nitta K., Yokokura T., Sawada S., Kunimoto T., Tanaka T., Uehara N., Baba H., Takeuchi M., Miyasaka T., Mutai M., Jpn. J. Cancer Chemother., 14, 850-857 (1987).

8) Furuta T., Yokokura T., Mutai M., Jpn. J. Cancer Chemother, 15, 2757-2760 (1988)

9) Furuta T., Yokokura T., Jpn. J. Cancer Chemother, 15, 2757-2760 (1990).

10) Araki E., Ishikawa M., Iigo M., Koide T., Itabashi M., Hoshi A., Jpn. J. Cancer Res., 84, 697-702 (1993).

11) Takasuna K., Hagiwara T., Hirohashi M., Kato M., Nomura M., Nagai E., Yokoi T., Kamataki T., Cancer Res., 56, 3752-3757 (1996).

12) Mick R., Gupta E., Vokes E. E., Ratain M. J., J. Clin. Oncol., 14, 2012-2019 (1996)

13) Machida Y., Onishi H., Morikawa A., Machida Y., S.T.P. Pharma Sci., 8, 175-181 (1998).

14) Machida Y., Onishi H., Kurita A., Hata H., Morikawa A., Machida Y., J. Control. Release, 66, 159-175 (2000).

15) Yokoyama M., Drug Delivery System, 14, 449—457 (1999).

16) Dunn S. E., Coombes A. G. A., Garnett M. C., Davis S. S., Davies M. C., Illum L., J. Control. Release, 44, 65-76 (1997).

17) Takakura Y., Takagi A., Hashida M., Sezaki H., Pharm. Res., 4, 293300 (1987).

18) Matsumura Y., Maeda H., Cancer Res., 46, 6387-6392 (1986). 